\title{
Composite Indicators for Data Mining: A New Framework for Assessment of Prediction Classifiers
}

\author{
Shahid Anjum
}

\begin{abstract}
Effectiveness and superiority of predictive accuracy of different Data mining (DM) models over the others have traditionally come from results of the empirical studies of DM. Study [4] compared logistic regression, classification tree, neural network, random forest and AdaBoost based on evaluation composite indicators (ECI) built from four parameters like accuracy, interpretability, robustness and speed using four input alternatives (original, aggregated, principal component analysis and stacking based variables), three random indicator weighting criteria and two indicator normalization methods (z-score and min-max). In this study, ECI has been calculated using results from [4] from same four input variable types but using "four plus one" (five) parameters. The fifth parameter of interest (POI) named as Residual Efficiency (RE), has been quantified for this study based on characteristics of interest (COI) described in [10]. Besides, analytical hierarchy process (AHP) of [13] has been used as weighting criteria and step wise utility functions of [12] as normalization technique. Finally we have compared our results with that of [4]. As opposed to study [4], this study has calculated ECIs for all the classifiers used and results have narrower ranges thus are more realistic for comparing the considered classifiers objectively based on type of inputs and POIs.
\end{abstract}

Index Terms-Knowledge discovery and data mining (KDD), analytical hierarchy process (AHP), evaluation composite indicators (ECI), multi-criteria decision making (MCDM).

\section{INTRODUCTION}

Data mining (DM) and knowledge discovery in databases (KDD) has been applied to a variety of application domains. Classification methods of data mining has been applied to understand the root causes of Asian financial crisis (twin banking and currency crisis of 1998) [1], credit scoring and evaluation, bankruptcy prediction, insurance underwriting, fraud detection, financial performance prediction, bond rating analysis, credit risk assessment, to forecast daily changes in seven financial stocks' prices [2] and other applications in finance [3]. Other important applications cited in this study are to customer churn prediction problem [4] and comparison of social objectives for decision-making in housing corporations [5].

Data modeling or building a model from data is what data mining techniques generate. There are many different data

Manuscript received March 18, 2013; revised June 10, 2013. Views expressed in this paper and all errors and emissions are that of the author and in no case reflect those of LTU or its management.

S. Anjum is with Faculty member (Finance and MIT) for Doctor of Management in Information Technology (DMIT) and Doctor of Business Administration (DBA) programs at College of Management, Lawrence Technological University (LTU), Buell Bldg., M331, 21000 West Ten Mile Road, Southfield, MI 48075-1058, USA (e-mail: anjumsw@ hotmail.com). mining algorithms with different objectives, different outcomes and with different representation techniques [6]. Data mining taxonomy includes predictive models and descriptive models. Descriptive models include association and clustering and predictive models include classification and regression. Predictive models can be regressor or classifiers [7]. Prediction techniques are similar to classification where unseen data is used to predict the class label of each row of data [8]. Simple parametric to nonparametric statistical methods are used to develop classifiers which are most commonly implemented with neural network (NN), decision tree (DT), Naïve Bayes, logistic regression (LR) or k-nearest- neighbor algorithms. Regarding assessment of classifier, most of comparisons have come from empirical literature where different classifiers were used as a solution approach for a particular problem setting as in [2] and various others (omitted to contain the reference list). Comparisons based on empirical studies reveal that there is no objective conclusion about superiority of one classifier over the other based on types of inputs used and parameters other than accuracy only. Performance of any classifier rather depends on the nature of problem, type of dataset to be used and behavior of variables in that particular problem and has been ranked based on accuracy parameter only in most cases.

\section{Literature REVIEW AND DATA DESCRIPTION}

Besides DM literature cited above, application of certain DM method has been backed by other multi-criteria decision making (MCDM) techniques like Analytical Hierarchy Process (AHP) etc. The use of AHP in a multiplicity of environments is well documented and studies like [3], [8] and [9], besides others, have used AHP in the context of DM. However, all these studies have used AHP for input variable selection. In literature, however, not various studies have been found which have attempted to compare the data mining models based on other discriminate parameters than accuracy only. The study [4] is the one which has attempted a comparison of classifiers by building evaluation composite indicators (ECIs) based on four parameters of Interest (POI) or assessment criteria (AC) in customer churn prediction problem.

In order to rank the DM techniques for classification, various authors have mentioned three POI including accuracy (A), interpretability (I) (or complexity) and speed (S) [7] and ref. [8] has added robustness (R) (or stability or consistency) and lift. The study [10] has quoted that in medical literature, validation ratios for classification algorithm (percentage of correct classified (PCC) for overall 
accuracy, sensitivity (true positive rate) and specificity (true negative rate) can be calculated from confusion matrix for a binary classification problem. Receiver operating characteristic (ROC) curve is a commonly used summary for assessing the tradeoff between sensitivity and specificity. It is a plot of the sensitivity versus specificity as we vary the parameters of a classification rule. The Area Under (ROC) Curve (AUC) or c-statistic is a commonly used quantitative summary for accuracy.

This study uses five POIs to arrive at final calculations of ECIs. Four POIs are same as in the reference study [4], which are A (=AUC), I, R (=AUCtest - AUCtrian), and S (or execution time). Here AUCtest (AUCtrain) means AUC for test (train) data. 'I' of individual classifiers has been defined in ref. [4] on a four point scale based on four categories for null, poor, medium and high interpretability with respective scores of 1, 2, 3 and 4. Based on this yardstick, classification tree (DT) have got high, logistic regression (LR) medium, neural network (NN), AdaBoost (AB) and random forest (RF) have received poor and stacking methodology and PCA have received null category scores. POIs are different from measures of interestingness (MOI) cited in study [6] describing taxonomy of MOI as objective (coverage, support, accuracy) and subjective (unexpected, actionable, novel). Third one is semantics-based MOI.

TABLE I: INFORMATION GATHERED ABOUT COI TO ARRIVE AT INDIVIDUAL PARAMETERS RESULTS (WITHOUT NORMALIZATION) OF RF-IVSO PAIRS FOR RESIDUAL EFFICIENCY PARAMETER

\begin{tabular}{|c|c|c|c|c|}
\hline \multirow{2}{*}{ COIs } & \multicolumn{5}{|c|}{ RF-IVSO Pairs } \\
\cline { 2 - 5 } & RF/OV & RF/AV & RF/PC & RF/SV \\
\hline COI-1 & 5 & 5 & 5 & 6 \\
\hline COI-2 & 6 & 6 & 6 & 6 \\
\hline COI-3 & 5 & 5 & 5 & 4 \\
\hline COI-4 & 6 & 6 & 6 & 6 \\
\hline COI-5 & 5 & 5 & 5 & 6 \\
\hline COI-6 & 6 & 6 & 6 & 6 \\
\hline COI-7 & 6 & 6 & 6 & 6 \\
\hline COI-8 & 6 & 6 & 6 & 6 \\
\hline COI-9 & 6 & 5 & 6 & 6 \\
\hline COI-10 & 5 & 5 & 5 & 6 \\
\hline
\end{tabular}

Note: (1) Explanation for cell value of COI-10 versus RF/OP2-SV pair means that because RF is an ensemble method (i.e. present) so it got a score of 3 and as stacking VSO in OP2-SV is also an ensemble method (i.e. present) so it also got a score of 3 and thus the value in respective cell is 6 :) (2) Information in this table has been compiled from these references [4], [6], [10], [9], and [11]

This study has developed a new POI, which has been named as Residual Efficiency (RE), whose concept is based on characteristics of interest (COI) for off-the-shelf (OTS) method [10]. RE is based on characteristics of interest (COI) that can make a method or limit it from being an "off-the-shelf (OTS) method" as described in ref. [10]. These COI include computational considerations, handling of messy data, missing values, long-tailed and skewed distributions of numeric predictor and response variables, mis-measurement (or outliers), handing of non-linearity, over-fitting issue, level of user interaction, scalability, sensitivity to monotone transformation of data or whether method is an ensemble method or not. We have used ten COI in order to arrive at RE. These are whether or not, any classifier or IVSO can handle non-linearity, don't have over-fitting problem, require low level of user interaction, is scalable (can handle large database), natural handling of mixed type data, can handle of outliers, insensitive to monotone transformation of data, ability to extract linear combination of the features, can handle noisy or missing data and is an ensemble method (represented by symbols from COI -1 to COI-10 respectively for these COI). In order to arrive at RE, we have tallied each COI for each classifier-IVSO pairs. Each COI can be in one of the either 'present', 'absent' or 'not applicable' state (with respective scores of 3, 2, and 1 for these states) for each classifier-IVSO pair. Table I describes information on all COI used for RE of RF-IVSO pairs i.e. for combinations of Random Forest classifier and four IVSO. The information on these COI have been gathered from various sources ([4], [6], [9], and [11]). The results for COI for RE (without normalization) have been provided in Table II.

TABLE II: RESULTS (WITHOUT NORMALIZATION) OF RESIDUAL EFFICIENCY (RE) POI FOR CLASSIFIER-VSO PAIRS

\begin{tabular}{|c|c|c|c|c|}
\hline & OV & AV & PCA & SV \\
\hline LR & 52 & 51 & 52 & 54 \\
\hline DT & 54 & 53 & 54 & 56 \\
\hline NN & 50 & 49 & 50 & 52 \\
\hline AB & 55 & 54 & 55 & 57 \\
\hline RF & 56 & 55 & 56 & 58 \\
\hline
\end{tabular}

Value of 58 in last cell is sum of column RF/OP2-SV in Table I (above)

The customer churn study [4] (reference study for this article) has used data about demographics of customer, purchasing profile and transactions and has applied four different input variable selection options (IVSO) or alternatives which has been labeled as OP1-OV (or OV) which kept 462 original variables, OP2-AV (or AV) which is composed of 584 variables, original and aggregates, OP3-PCA (or PC) which has 184 selected factors from AV using principal component analysis and OP2-SV (or SV) which has only 17 variables selected using stacking. In this article, we have used all the scores of ref. [4] and have combined those with RE, our fifth POI, and have given weights to all these five POIs using AHP.

\section{Methodology}

Five DM classifiers (LR, T, NN, AB and RF) has been combined with four IVSOs used in study [4] to rank different "DM classifiers-IVSO" pairs based on four POIs, using three POI weighting criteria (WC). First criteria provides equal weights to all four POIs, second gives $34 \%$ weight to A and $22 \%$ to rest of three (I, R \& S) and third criteria assigns $30 \%$ weights to A \& I and $20 \%$ to S \& R. The study [4] has used two indicator normalization methods (NM) which are z-score (z) and min-max (mm), in an effort to make differences in units of measurements (UOM) of POIs to disappear.

In this article, we have included five POIs which are A, I, $\mathrm{R}, \mathrm{S}$ and RE to arrive at ECIs for same five classifiers and four IVSO combination pairs. RE, our new POI, is not data or problem dependent thus inclusion of fifth POI will still pose 
no comparability issues with the results of study [4]. And Tables III and IV provide the results (without normalization) for various pairs of Classifiers-IVSOs of four POI (A, I, R, S) that has been borrowed from study [4]. The results in Tables III and IV will be used with our fifth POI (Table II) to arrive at new ECI measures in this study.

TABLE III: RESULTS (WITHOUT NORMALIZATION) OF VARIOUS PAIRS OF CLASSIFIERS-IVSO FOR

\begin{tabular}{|c|c|c|c|c|c|c|c|c|}
\hline \multirow{2}{*}{} & \multicolumn{4}{|c|}{ Accuracy } & \multicolumn{4}{c|}{ Speed (Minutes) } \\
\cline { 2 - 10 } & OV & AV & PC & SV & OV & AV & PC & SV \\
\hline LR & 0.80 & 0.80 & 0.80 & 0.79 & 18 & 21 & 04 & $215+1$ \\
\hline DT & 0.77 & 0.78 & 0.66 & 0.79 & 05 & 05 & 02 & $215+1$ \\
\hline NN & 0.80 & 0.77 & 0.56 & 0.82 & 05 & 05 & 02 & $215+1$ \\
\hline AB & 0.73 & 0.77 & 0.65 & - & 13 & 20 & 06 & - \\
\hline RF & 0.79 & 0.81 & 0.68 & - & 06 & 70 & 16 & - \\
\hline
\end{tabular}

Note: For explanation see study [4]

TABLE IV: RESULTS (WITHOUT NORMALIZATION) OF VARIOUS PAIRS OF CLASSIFIERS-IVSOS FOR

\begin{tabular}{|c|c|c|c|c|c|c|c|c|}
\hline & \multicolumn{4}{|c|}{ Robustness } & \multicolumn{4}{c|}{ Interpretability } \\
\hline & OV & AV & PC & SV & OV & AV & PC & SV \\
\hline LR & 0.04 & 0.05 & 0.04 & 0.08 & 3 & 3 & 1 & 1 \\
\hline DT & 0.04 & 0.03 & 0.06 & 0.07 & 4 & 4 & 1 & 1 \\
\hline NN & 0.03 & 0.03 & 0.03 & 0.04 & 2 & 2 & 1 & 1 \\
\hline AB & 0.16 & 0.16 & 0.15 & - & 2 & 2 & 1 & - \\
\hline RF & 0.01 & 0.00 & 0.00 & - & 2 & 2 & 1 & - \\
\hline
\end{tabular}

Note: For explanation see study [4]

Three value additions of this study as compared to study [4] can be described as. The calculation of RE (Table II) from ten COIs is the one. Use of a different UOM normalization technique for POIs, a stepwise utility function (SWUF as called here) of study [12], is the second one. Third value addition is that this article has used AHP as weighting criterion for POIs instead of randomly selected three criteria used by study [4].

AHP is a type of MCDM method where judgmental inputs and solution outputs are scalar and fixed point values and which mostly uses mathematical deterministic solution procedures. AHP involves decomposing the problem into a hierarchy, assessing the normalized relative importance weights $\left[\mathrm{W}=\left(w_{1} \ldots . . w_{\mathrm{m}}\right)^{\mathrm{t}}\right]$ of $P O I_{1}, \ldots, P O I_{\mathrm{m}}$ decision criteria using pair wise comparisons (PWC) that are quantified using Saaty's 1-9 points scale (see [13]) which satisfies the normalization condition of $\Sigma_{\mathrm{j}=1 \ldots \mathrm{m}}\left[w_{\mathrm{j}}\right]=1$ with $w_{\mathrm{j}}>=0$ for $\mathrm{j}=1, \ldots, \mathrm{m}$. Based on PWC scale in conventional AHP, POI $_{i}$ can be equally important, moderately more important, strongly more important, very strongly more important or extremely strongly more important than $\mathrm{POI}_{\mathrm{j}}$ and thus will receive 1, 3, 5, 7 or 9 score on Saaty's gradation scale respectively [14].

Main advantage of PWC is the easiness of comparing two items at a time than to compare many items all at once. And information guiding these PWCs can come from literature. For example, regarding interpretability, study [7] describes that predictive model's descriptive aspect (i.e. interpretability) is even a more important POI than its ability to predict. Study [4] has chosen, while defining POI weights, only accuracy (A) with $34 \%$ weight as compared to rest three

(I, R \& S) with $22 \%$ weights emphasizing that accuracy is important than all three other POIs used in the study. Same study in its third weighting criteria has assigned $30 \%$ weights to A \& I and $20 \%$ to S \& R meaning that 'I' is another POI which is more important than other two (R \& S) and equally important to accuracy. Besides decision maker can, based on the information about the type of hardware used by an organization, type of distributive computing model used (i.e. in-house or clouding), type of dataset and focus of the study, decide about the PWC ratios for speed versus robustness. Table V shows our PWC matrix for various POIs.

TABLE V: AHP BASED PWC MATRIX OF POIS
\begin{tabular}{|l|c|c|c|c|c|}
\hline & A & I & R & S & RE \\
\hline A & 1 & 3 & 5 & 7 & 9 \\
\hline I & $1 / 3$ & 1 & 3 & 5 & 7 \\
\hline $\mathbf{R}$ & $1 / 5$ & $1 / 3$ & 1 & 5 & 5 \\
\hline S & $1 / 7$ & $1 / 5$ & $1 / 5$ & 1 & 5 \\
\hline RE & $1 / 9$ & $1 / 7$ & $1 / 5$ & $1 / 5$ & 1 \\
\hline
\end{tabular}

We will use principal right eigenvector method (EM) as in [15] to solve AHP matrix. EM involves the determination of weight vector (W) from the PWC matrix (Table V) by solving the characteristic equation $\mathrm{AW}=\lambda_{\max } \mathrm{W}$; where $\lambda_{\max }$ is the maximum eigen-value of A (=PWC Matrix). Three issues surround the use of the AHP [16]. The first issue is inconsistency problem and it occurs because the allowable upper bound of consistency index (CI) is $10 \%$ of the random inconsistency (RI). Consistency in PWC Matrix can be checked by the consistency ratio $(\mathrm{CR})$ which is equal to $\left\{\left[\left(\lambda_{\max }-1\right) /(n-1)\right] / R I\right\}$. Here RI's value varies with the order of PWC matrix. A CR of less than or equal to $10 \%$ is considered of an acceptable consistency. The second issue is rank-reversal problem [18] of range of $w_{\mathrm{ij}}$, the relative weight of alternative $i$ to $j$. There are total of 20 alternatives (Classifier-IVSO pairs) in our case.

Third issue is discriminating-sensitivity problem i.e. if the range of $\mathrm{w}_{\mathrm{ij}}$ were to be too reduced, and then CI would converge to 0 , making it impossible for the AHP to discriminate an important alternative from others. This can be avoided by using Saaty's1-9 scale for PWCs. Our calculated $\mathrm{CR}$ (=CI/index of consistency) is $10 \%$. In short, our comparisons are consistent and there is no question of discriminating - sensitivity problem as well.

The objective of AHP is to compare decision alternatives (i.e. 20 Classifier-IVSO pairs) with respect to each POI and to determine the relative composite priorities for the true total weights of Classifier-IVSO pairs, when the POIs are assembled together. Our solution to our pair wise matrix gives us weights for various POI as: 0.498531 for A, 0.256196 for I, 0.148403 for R, 0.066924 for $\mathrm{S}$ and 0.029945 for RE. But as POIs have different dimensions or UOM, there is a problem of incommensurability. We can address the issue of differences in dimensions through normalization.

To effectively deal with incommensurability, we have chosen a SWUF described in [12]. In contrast to the min-max normalization procedure used by study [4] which rewarded and/or penalized all values, we have used SWUF (from [12]) for normalization for UOM of POI. It has applied points scoring system for POI to measure the contribution of intervals performance of each POI. It has used a 0-10 scale with 6 intervals which have been defined with $0,2,4,6,8$, 
and 10 points respectively (as shown in Table VI). The intermediate intervals have equal width, which implies that essentially they are assuming a linear relationship.

TABLE VI: RULES FOR POI SCORES FOR SWUF OF REF. [12]

\begin{tabular}{|c|c|c|c|c|c|}
\hline $\begin{array}{l}\text { Scores } \\
\text { (below) }\end{array}$ & $\overline{\mathbf{A}}$ & I & $\mathbf{R}$ & $\mathbf{S}$ & RE \\
\hline 0 & If $<0.65$ & Null $=1$ & $\begin{array}{l}\text { If > } \\
0.19\end{array}$ & $\begin{array}{l}\text { If }> \\
100\end{array}$ & $\begin{array}{c}\text { If }< \\
50\end{array}$ \\
\hline 2 & $\begin{array}{c}\text { If } \\
0.65-0.68\end{array}$ & $\begin{array}{l}\text { Null } \\
=1\end{array}$ & $\begin{array}{c}\text { If } 0.16 \\
-0.19\end{array}$ & $\begin{array}{c}\text { If } 25- \\
100\end{array}$ & $\begin{array}{c}\text { If } 50 \\
-51\end{array}$ \\
\hline 4 & $\begin{array}{c}\text { If } \\
0.69-0.72\end{array}$ & $\begin{array}{c}\text { Poor }= \\
2\end{array}$ & $\begin{array}{l}\text { If } 0.12 \\
-0.15\end{array}$ & $\begin{array}{c}\text { If } 19- \\
24\end{array}$ & $\begin{array}{c}\text { If } 52 \\
-53\end{array}$ \\
\hline 6 & $\begin{array}{c}\text { If } \\
0.73-0.76\end{array}$ & $\begin{array}{c}\text { Poor }= \\
2\end{array}$ & $\begin{array}{l}\text { If } 0.08 \\
-0.11\end{array}$ & $\begin{array}{c}\text { If } 12- \\
18\end{array}$ & $\begin{array}{c}\text { If } 54 \\
-55\end{array}$ \\
\hline 8 & $\begin{array}{c}\text { If } \\
0.77-0.80\end{array}$ & $\begin{array}{c}\text { Mediu } \\
\mathrm{m}=3\end{array}$ & $\begin{array}{l}\text { If } 0.04 \\
-0.07\end{array}$ & $\begin{array}{c}\text { If } 6- \\
11\end{array}$ & $\begin{array}{c}\text { If } 56 \\
-57\end{array}$ \\
\hline 10 & If $>0.80$ & $\begin{array}{c}\text { High } \\
4\end{array}$ & $\begin{array}{l}\text { If }< \\
0.04\end{array}$ & If $<6$ & $\begin{array}{c}\text { If }> \\
57\end{array}$ \\
\hline
\end{tabular}

(1). Entry " $50-51$ " means that if values of this POI vary from 50 to 51 for RE, then its score will be 2 .

(2). [Poor $=2]$ will get score of 6 if both components are linear in the classifier-IVOR pair and [Poor=2] will get score of 4 if one of the components is linear and another nonlinear in the classifier-IVOR pair (3) [Null = 1] will get score of 2 if both components are linear in the classifier-IVOR pair and [Null $=1]$ will get score of 0 if one of the components is linear and another nonlinear in the classifier-IVOR pair.

General considerations for our POI can be described as follow. For accuracy (AUC test), larger value is considered better than smaller value. Similar is the case for two other POI, interpretability, and residual efficiency. On the other hand for robustness and speed, lower value is better than higher one. Individual parameters results of Tables II, III \& IV has been normalized with SWUF of ref. [12] for various pairs of Classifiers-IVSO for all five POI (A, I, R, S and RE) and presented in Tables VII, VIII, IX and 10. The explanation of these three tables can be seen in study [4] and if some cell(s) has got zero value in all three tables, it has been replaced with a value of 0.0001 , so as to avoid making the weight of respective POI zero. And this small adjustment had no affect on relative weights of POIs.

TABLE VII: INDIVIDUAL PARAMETERS RESULTS (NORMALIZED WITH SWUF) OF VARIOUS PAIRS OF CLASSIFIERS-IVSO FOR ROBUSTNESS AND INTERPRETABILITY

\begin{tabular}{|c|c|c|c|c|c|c|c|c|}
\hline & \multicolumn{4}{|c|}{ Robustness } & \multicolumn{4}{|c|}{ Interpretability } \\
\hline & OV & AV & PC & SV & OV & AV & PC & SV \\
\hline LR & 8 & 8 & 8 & 6 & 8 & 8 & 2 & 0 \\
\hline DT & 8 & 10 & 8 & 8 & 10 & 10 & 2 & 0 \\
\hline NN & 10 & 10 & 10 & 8 & 4 & 4 & 0 & 0 \\
\hline AB & 2 & 2 & 4 & 0 & 4 & 4 & 0 & 0 \\
\hline RF & 10 & 10 & 10 & 0 & 4 & 4 & 0 & 0 \\
\hline
\end{tabular}

TABLE VIII: INDIVIDUAL PARAMETERS RESULTS (NORMALIZED WITH SWUF) OF VARIOUS PAIRS OF CLASSIFIERS-IVSO FOR ACCURACY AND SPEED POIS

\begin{tabular}{|c|c|c|c|c|c|c|c|c|}
\hline & \multicolumn{4}{|c}{ Accuracy (AUC Test) } & \multicolumn{4}{c|}{ Speed (Minutes) } \\
\hline & OV & AV & PC & SV & OV & AV & PC & SV \\
\hline LR & 8 & 8 & 8 & 8 & 6 & 4 & 10 & 0 \\
\hline DT & 8 & 8 & 2 & 8 & 10 & 10 & 10 & 0 \\
\hline NN & 8 & 8 & 0 & 8 & 10 & 10 & 10 & 0 \\
\hline AB & 6 & 8 & 2 & 0 & 6 & 4 & 8 & 0 \\
\hline RF & 8 & 1 & 2 & 0 & 8 & 2 & 6 & 0 \\
\hline
\end{tabular}

TABLE IX: INDIVIDUAL PARAMETERS RESULTS (NORMALIZED WITH SWUF) OF VARIOUS PAIRS OF CLASSIFIERS-IVSO FOR RESIDUAL EFFICIENCY (RE)

\begin{tabular}{|c|c|c|c|c|}
\hline & OP1-OV & OP2-AV & OP3-PCA & OP2-SV \\
\hline LR & 4 & 2 & 4 & 6 \\
\hline DT & 6 & 4 & 6 & 8 \\
\hline NN & 2 & 0 & 2 & 4 \\
\hline AB & 6 & 6 & 6 & 8 \\
\hline RF & 8 & 6 & 8 & 10 \\
\hline
\end{tabular}

TABLE X: FINAL ECI VALUES FOR AHP WEIGHTING AND NORMALIZATIONS WITH SWUF (IN \%)

\begin{tabular}{|c|c|c|c|c|}
\hline & OV & AV & PC & SV \\
\hline LR & $8.175 \%$ & $8.001 \%$ & $6.008 \%$ & $4.464 \%$ \\
\hline DT & $9.267 \%$ & $9.422 \%$ & $3.320 \%$ & $4.733 \%$ \\
\hline NN & $6.964 \%$ & $6.907 \%$ & $1.704 \%$ & $4.620 \%$ \\
\hline AB & $5.079 \%$ & $5.876 \%$ & $1.978 \%$ & $0.226 \%$ \\
\hline RF & $7.016 \%$ & $3.406 \%$ & $2.553 \%$ & $0.283 \%$ \\
\hline
\end{tabular}

ECI values from our methodology i.e. using five POI, using AHP for weighting and UOM normalizations with SWUF (in Percentages) has been given in Tables XI (a-d). Finally results for four POI used by ref. [4] with min-max and z-score normalization schemes and three weighting methods has been compared with this article's exclusive SWUF normalization coupled with AHP weighting has been provided side by side in Tables XI (a-d). Results from ref. [4] show that for OV, ranking of DM classifiers based on ECI is that $\mathrm{T}$ is first, LR second, NN is third, RF is fourth and AB is last for all of different weighting alternatives $(1,2 \& 3)$ and two normalizations schemes ( $\mathrm{z} \& \mathrm{~mm})$ i.e. as shown under labels of ECImm\&1, ECImm\&2, ECImm\&3, ECIz\&1, ECIz\&2, ECIz\&3. On the other hand, in our calculation, all other positions are same except that RF has gained third position and NN as fourth by a very small margin (Table XI A). For AV, T is first, LR second, RF is third, NN is fourth and $\mathrm{AB}$ is last for all of different weighting alternatives and normalizations (i.e. ECImm\&2, ECImm\&3, ECIz\&1, ECIz\&2, ECIz\&3) of study [4] except ECImm\&1. While in our calculation, first two positions remained same and $\mathrm{NN}$ as third, $\mathrm{AB}$ as fourth and $\mathrm{RF}$ has gained last position. Ranking of DM classifiers for PC, results are that LR is first, RF is second, T is third, AB is fourth and NN is last for ECImm\&2, ECImm\&3, ECIz\&1, ECIz\&2, ECIz\&3 used by study [4] except for ECImm\&1 where NN got fourth and $\mathrm{AB}$ last position. While in our calculation, $\mathrm{LR}$ became first and $\mathrm{T}$ is second, RF is third, $\mathrm{AB}$ is fourth and $\mathrm{NN}$ has gained last position. For $\mathrm{SV}, \mathrm{NN}$ is first, $\mathrm{T}$ as second and $\mathrm{LR}$ is third for ECImm\&1, ECImm\&2, ECImm\&3, ECIz\&1, ECIz\&2, ECIz\&3 used by study [4] while AB and RF were not ranked. While in our calculation, T became first, NN as second, LR as third, RF as fourth and AB has gained last position. However, important to note is that our method has ranked even $\mathrm{AB}$ and RF successfully and logically for all entries in contrast to study [4] where last two rows were not ranked. 
TABLE XI A: ECI VALUES FOR THE DIFFERENT NM AND WC FOR OV

\begin{tabular}{|c|c|c|c|c|c|}
\hline & LR & DT & NN & AB & RF \\
\hline ECImm\&1 & 0.326 & 0.383 & 0.269 & 0.039 & 0.230 \\
\hline ECImm\&2 & 0.402 & 0.434 & 0.347 & 0.135 & 0.311 \\
\hline ECImm\&3 & 0.423 & 0.488 & 0.341 & 0.149 & 0.308 \\
\hline ECIz\&1 & 0.551 & 0.667 & 0.388 & -0.356 & 0.291 \\
\hline ECIz\&2 & 0.565 & 0.599 & 0.406 & -0.288 & 0.314 \\
\hline ECIz\&3 & 0.587 & 0.717 & 0.351 & -0.277 & 0.268 \\
\hline SWUF\&AHP & $\mathbf{0 . 0 8 1 7}$ & $\mathbf{0 . 0 9 2 7}$ & $\mathbf{0 . 0 6 9 6}$ & $\mathbf{0 . 0 5 0 8}$ & $\mathbf{0 . 0 7 0 2}$ \\
\hline
\end{tabular}

TABLE XI B: ECI VALUES FOR THE DIFFERENT NM AND WC FOR AV

\begin{tabular}{|c|c|c|c|c|c|}
\hline & LR & DT & NN & AB & RF \\
\hline ECImm\&1 & 0.307 & 0.417 & 0.241 & 0.023 & 0.238 \\
\hline ECImm\&2 & 0.384 & 0.472 & $\mathbf{0 . 3 1 1}$ & 0.121 & $\mathbf{0 . 3 2 5}$ \\
\hline ECImm\&3 & 0.407 & 0.521 & $\mathbf{0 . 3 0 8}$ & 0.136 & $\mathbf{0 . 3 2 0}$ \\
\hline ECIz\&1 & 0.490 & 0.786 & $\mathbf{0 . 2 8 1}$ & -0.404 & $\mathbf{0 . 3 3 1}$ \\
\hline ECIz\&2 & 0.507 & 0.733 & $\mathbf{0 . 2 6 3}$ & -0.329 & $\mathbf{0 . 3 7 6}$ \\
\hline ECIz\&3 & 0.535 & 0.837 & $\mathbf{0 . 2 2 4}$ & -0.314 & $\mathbf{0 . 3 2 2}$ \\
\hline SWUF\&AHP & $\mathbf{0 . 0 8 0 0}$ & $\mathbf{0 . 0 9 4 2}$ & $\mathbf{0 . 0 6 9 1}$ & $\mathbf{0 . 0 5 8 8}$ & $\mathbf{0 . 0 3 4 1}$ \\
\hline
\end{tabular}

TABLE XI C: ECI VALUES FOR THE DIFFERENT NM AND WC FOR PC

\begin{tabular}{|c|c|c|c|c|c|}
\hline & LR & DT & NN & AB & RF \\
\hline ECImm\&1 & 0.168 & 0.006 & -.042 & -0.076 & 0.102 \\
\hline ECImm\&2 & 0.259 & 0.053 & $\mathbf{- 0 . 0 3 7}$ & 0.014 & 0.146 \\
\hline ECImm\&3 & 0.227 & 0.045 & $\mathbf{- 0 . 0 3 3}$ & 0.007 & 0.129 \\
\hline ECIz\&1 & 0.098 & -0.518 & $\mathbf{- 0 . 7 4 4}$ & -0.725 & -0.191 \\
\hline ECIz\&2 & 0.151 & -0.638 & $\mathbf{- 1 . 0 2 5}$ & -0.691 & -0.315 \\
\hline ECIz\&3 & 0.025 & -0.673 & $\mathbf{- 1 . 0 1 1}$ & -0.731 & -0.382 \\
\hline SWUF\&AHP & $\mathbf{0 . 0 6 0 1}$ & $\mathbf{0 . 0 3 3 2}$ & $\mathbf{0 . 0 1 7 0}$ & $\mathbf{0 . 0 1 9 8}$ & $\mathbf{0 . 0 2 5 5}$ \\
& & & & & \\
\hline
\end{tabular}

TABLE XI D: ECI VALUES FOR THE DIFFERENT NM AND WC FOR SV

\begin{tabular}{|c|c|c|c|c|c|}
\hline & LR & DT & NN & AB & RF \\
\hline ECImm\&1 & -0.143 & -0.129 & -0.057 & - & - \\
\hline ECImm\&2 & -0.019 & -0.006 & 0.070 & - & - \\
\hline ECImm\&3 & -0.025 & -0.014 & 0.054 & - & - \\
\hline ECIz\&1 & -0.970 & -0.739 & -0.490 & - & - \\
\hline ECIz\&2 & -0.891 & -0.600 & -0.330 & - & - \\
\hline ECIz\&3 & -0.914 & -0.656 & -0.415 & - & - \\
\hline SWUF\&AHP & $\mathbf{0 . 0 4 4 6}$ & $\mathbf{0 . 0 4 7 3}$ & $\mathbf{0 . 0 4 6 2}$ & $\mathbf{0 . 0 0 2 3}$ & $\mathbf{0 . 0 0 2 8}$ \\
\hline
\end{tabular}

The results (in Tables XI A-XI D) have shown that our method has proven to be robust on following grounds. First of all it has not generated any negative entries. Secondly, contrary to the results from study [4] which have kept almost the same hierarchy for all classifiers with only few minor exceptions, results of this study has not followed any specific pattern regarding ranking for all classifiers as our results has provided a different ranking of DM classifiers for all four different types of input variables. And this is important because performance of data mining models is dependent on factors like problem in hand, type of dataset, depth of database, types and nature of relationships among input variables and/or target variables. Thus ranking of DM models should not be considered permanent and should change as these factors change. This is, however, different from objective ranking drawn for various DM models from the broader categories of inputs used, as has been attempted here. Last but not least, our method was able to rank all the classifiers for all variable types as opposed to study [4] who was not able to rank $\mathrm{AB}$ and RF for VS.

In short, the new methodology using AHP as weighting the assessment criteria or POI and with SWUF as normalization technique to tackle the issue of incommensurability because of difference in UOM for POI (or criteria) has worked well in ranking data mining algorithms. Another main contribution of the paper was the development of a fifth POI (named as $\mathrm{RE}$ ) from ten characteristics of interest (COI) derived from vast body of data mining literature, have worked very well in ranking the data mining algorithms and the robustness of our results are deeply indebted to this fifth POI.

\section{REFERENCES}

[1] S. Anjum, "Early warning system for financial crisis: a critical review and application of data mining approach," Ph. D. Dissertation, GSID, Nagoya University, Japan, March 2003.

[2] L. C. Tjung, O. Kwon, K. C. Tseng, and J. Bradley, "Forecasting financial stocks using data mining," Craig School of Business, CSU, Fresno, 2010.

[3] A. Nadali, S. Pourdarab, and H. E. Nosratabadi, "Labeling the class of bank credit's customers by a fuzzy expert system for credit scoring with data mining approach," in Proc. IEEE Knowledge Discovery Conference, 2011.

[4] M. Clemente, V. G. Bosch, and S. San Matías. (2010). Assessing classification methods for churn prediction by composite indicators. Manuscript. Dept. of Applied Statistics and Quality. Universitat Politècnica de València. Camino de Vera s/n. 46022 Spain. [Online]. Available:

http://www.upv.es/deioac/Investigacion/ManuscriptDSS.pdf

[5] B. Kramer, D. Kronbichler, T. V. Welie, and J. Putman, "Comparing social objectives for decision-making in housing corporations," Applied Working Paper, no. 2008-02, Ortec Finance Research Center (OFRC) working paper series, May 2008, Rotterdam.

[6] K. McGarry, "A survey of interestingness measures for knowledge discovery," The Knowledge Engineering Review, Cambridge University Press, pp. 1-24, 2005.

[7] R. Gerritsen, "Assessing loan risks: a data mining case study," IT Pro, IEEE, November/December, 1999.

[8] K. Niki and H. R. Weistroffer, "Expanding the knowledge base for more effective data mining," Manuscript, Virginia Commonwealth University, 1015 Floyd Avenue, School of Business, P. O. Box 844000-4000, Richmond VA, USA, 2005.

[9] J. Thongkam, G. Xu, and Y. Zhang, "AdaBoost algorithm with random forests for predicting breastcancer survivability," in Proc. Intl. Joint Conf. on Neural Networks (IJCNN 2008), IEEE, 2008.

[10] T. Hastie, R. Tibshirani, and J. Friedman, The elements of statistical learning: Data mining, inference, and prediction, Springer Series in Statistics, $2^{\text {nd }}$ ed., 2008.

[11] SAS, Data mining using as enterprise miner: A case study approach, SAS Publishing, 2003.

[12] Hemphill, L. J. Berry, and S. McGreal, "An indicator-based approach to measuring sustainable urban regeneration performance: Conceptual foundations and methodological framework," Urban Studies, vol. 414, ch. 1, pp. 725-755, 2004.

[13] T. L. Saaty, The Analytic Hierarchy Process, Mc Graw-Hill, NY, 1980.

[14] K. Al-Harbi and M. Al-Subhi, "Application of the AHP in project management," Intl. Journal of Project Management, vol. 19, pp. 19-27, 2001.

[15] S. I. Gass and T. Rapcsak, "Singular value decomposition in AHP," presented at International Symposium on the Analytic Hie-rarchy Process, Berne, Switzerland, August, 2001.

[16] Y. Sato, "The impact on scaling on the pair-wise comparison of the analytic hierarchy process," presented at International Symposium on the Analytic Hie-rarchy Process, Berne, Switzerland, August, 2001. 
Shahid W. Anjum is an adjunct faculty member (Finance and MIT), a member of dissertation committee for Doctor of Management Information Technology (DMIT) program and has been teaching Financial Valuation course to Doctor of Business Administration (DBA) program at College of Management, Lawrence Technological University (LTU) in USA, besides working for one of the leading financial institutions in Canada.
He has earned his $\mathrm{Ph}$. D. with dissertation focus on applications of data mining for the early warning system of financial crisis and banking and currency risks from GSID, Nagoya University (NU), Japan. He has also studied in Master of Computer Science program at Department of Mathematics and Computer Science, University of Detroit Mercy (UDM), USA. Besides he has earned M. Phil. Economics degree from QA University, Islamabad. He is member of Global Association of Risk Professionals (GARP) and Canadian Securities Institute (CSI). He has worked in Japan, USA, Canada, and as an intern at Asian Development Bank (ADB), Manila, Philippines. 